\title{
Le città nelle fortificazioni: le isole ioniche in Grecia. Conoscenza e valorizzazione di un patrimonio
}

The cities in the fortifications: the Ionian islands in Greece. Knowledge and enhancement of a heritage

\author{
Silvia Gron $^{\text {a }}$, Eleni Gkrimpa ${ }^{\text {b }}$ \\ ${ }^{\text {a }}$ Politecnico di Torino, Turin, Italy, silvia.gron@polito.it \\ b Aristotle University of Thessaloniki, Thessaloniki, Greece, elenigkrimpa@gmail.com
}

\begin{abstract}
Residing in the Mediterranean Sea, Ionian islands signify the passage from the west to the east. A constantly sought-after region due to the trade routes, was for a long time garrisoned and under the authority of the Venetian Republic (fourteenth-eighteenth centuries) that hindered with its fleet the Turkish invasions. The bigger islands that constitute the cluster of the Eptanisa: Corfu, Lefkada or Santa Maura, Ithaka, Kefalonia, Kythira, Zakinthos and more, that had strategic positions with respect to the usual routes, had since the middle ages fortifications like walls, towers and castles, that over the time were expanded and restructured by the Venetians in order to defend those islands from the enemy attacks. The rich iconographic historic material, considering the Ionian Islands, allows to document the characteristics of those wide spread defensive structures and to identify each strong part of this big and unique fortification cluster. It has to be noted that every one of those structures gives us clues about the urban history of the city it resides since they were part of the urban landscape revealing this way the urban layout. The compelling story of the architectural consistency of those fortresses, as it is described in the historic documents, cannot be always verified. Many of those structures are nowadays completely destroyed and only a few remains are left. There are many ways to organize a project for saving those structures and in particular one that will be related with the cultural tourism.
\end{abstract}

Keywords: Ionian islands, heritage network, preservation project, tourism.

\section{Introduzione}

L'arcipelago ionico è formato da numerose isole, sette le maggiori, suddivise in tre nuclei: quello a nord, disposto in corrispondenza dell'attuale confine fra Grecia e Albania, si compone delle isole di Corfù (K乏́

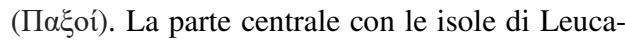

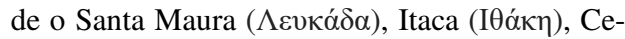

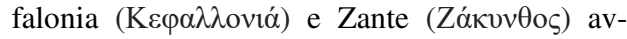
volge l'ingresso ai golfi di Patrasso e Corinto, mentre l'ultimo nucleo disposto verso l'isola di Creta, fra il mar Ionio ed Egeo, è quello compo- sto dall'isola di Cerigo (Kú $\theta \eta \rho \alpha)$. L'alleanza fra le diverse isole è tale da costituire un vero sistema a rete che si delinea con il dominio della Repubblica Veneziana (XIV-XVIII secolo), le isole quali terre di mare, sono fonte di proprie risorse in particolare per le saline e le piantagioni di uli$\mathrm{vi}$, ma risultano anche strategiche per la sorveglianza delle rotte fra occidente e oriente. È il commercio dell'olio a destare maggiore interesse per la Repubblica Veneziana, il suo impiego non è solo di tipo alimentare (consumo pari 
all'10\%), ma soprattutto produttivo, infatti il $40 \%$ viene destinato all'industria del sapone, utile a sua volta per la fase di "purgatura" nella lavorazione dei tessuti in lana. La confezione del sapone già nel Cinquecento "richiede un terzo di olio di oliva, si calcola che a quel tempo per ottenere 18000 libbre di buon sapone occorrevano oltre 6000 libbre d'olio" (Moroni, 2013, p. 140). Il commercio del sapone è considerato all'epoca fra quelli più remunerativi e Venezia lo esporta anche nel nord Europa (50\% della propria dotazione). La sempre più emergente richiesta di olio e i continui cambiamenti relativi alle dinamiche produttive nei territori del Mediterraneo, fa si che nel 1623 il governo veneziano attui una politica di implementazione delle coltivazioni d'ulivo che coinvolge le isole ioniche e nel contempo esegua controlli specifici sul traffico applicando dazi sul consumo d'olio (Ciriacono, 1997, p. 309). La produzione e il commercio sostengono l'interesse da parte dei Veneziani ad ampliare e ristrutturare le proprie fortezze cosa che capita per le isole ioniche già dotate di opere fortificate quali mura, torri, masti e castelli, in modo da meglio rispondere al controllo del territorio e agli attacchi nemici.

Dopo il lungo governo dei Veneziani l'arcipelago rimane un'entità solidale, infatti le isole del Levante con il Trattato di Campoformio nel 1797 saranno cedute ai Francesi per poi costituire la Repubblica Eptanese (nel periodo fra il 1800-1807) un nuovo governo greco semiautonomo ovvero dipendente dagli imperi russo e ottomano con capitale Corfù. Dopo una breve occupazione francese (1807-1809) e una inglese (1809-1815) sarà il Congresso di Vienna (18141815) ad assegnare le isole al prorettorato britannico sino al 1864 quando l'intero arcipelago costituirà definitivamente parte del territorio greco.

In questo contesto le fortificazioni risultano essere le opere di maggior importanza, non solo quali caposaldi di difesa o perché includono l'abitato ma soprattutto perché nodi del sistema infrastrutturale dell'intero territorio, nel definire spazi geografici caratterizzanti. Quasi la totalità di queste fortezze ora si trova allo stato di rudere, dove le tracce murarie solo in parte restituiscono la forma e la dimensione di un tempo.
Per svolgere alcune considerazioni sulle potenzialità attuali di questi siti è indispensabile ricostruirne la consistenza, per questo si esamina la documentazione iconografica seicentesca che illustra le strutture in epoca Veneziana, confrontandola poi alle campagne di rilievo e stato dei luoghi operate dai francesi (1798-1810), raccolta presso gli archivi militari nazionali, che illustra verosimilmente la distribuzione dell'edificato a conclusione del dominio veneziano. Le indagini eseguite convergono nel mostrare come modernizzare le opere o constatarne il degrado. Le mappe, planimetrie e sezioni quotate restituiscono così uno stato dell'arte delle opere, confrontabile con lo stato attuale nel definire una politica di salvaguardia e valorizzazione.

\section{Analisi del Patrimonio: le Fortezze delle Isole Ioniche}

Il patrimonio che si può iscrivere come Fortezze delle Isole Ioniche è molto vario e riguarda da piccoli castelli a vere e proprie città, questo coinvolge non solo le grandi isole ma anche le piccole e gli scogli, sono strutture o totalmente rifunzionalizzate come quella di Corfù, o ridotte a pochi ruderi oggi difficilmente individuabili. Un patrimonio oggetto di tutela da parte dell'Eforato -Ministero della Cultura e dello Sport- responsabile dello svolgimento delle indagini preliminari, della conservazione, restauro e valorizzazione del bene, garantendone anche la visita.

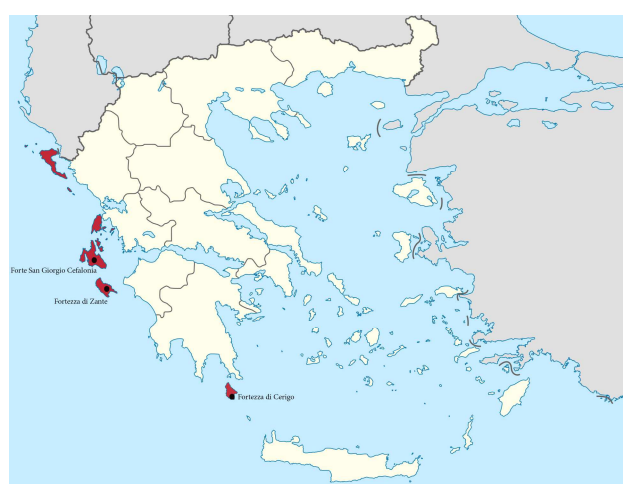

Fig. 1. Le isole ioniche e le fortezze analizzate.

La documentazione su queste Fortezze è ampia ma distribuita nei vari archivi degli Stati occu- 
panti, purtroppo mancano approfonditi studi sull'origine e le trasformazioni subite soprattutto nel definire un disegno d'insieme che possa valorizzarli. A titolo di esempio si analizzano tre casi disposti nelle isole grandi (Cefalonia, Zante, Cerigo) scelti perché, seppur in stato di abbandono, risultano meta di visita rientrando pertanto fra i siti di valore artistico/architettonico (Fig. 1).

\subsection{Forte San Giorgio Cefalonia (Kefalonia)}

Posto a sud-ovest dell'isola, nell'entroterra, a circa $5 \mathrm{Km}$ dal mare, in cima a una collina riparata dal Monte Nero, il Forte di S. Giorgio domina la piana di Argostoli (Fig. 2). Le sue origini sono antiche e lo mostra la sua conformazione che sfrutta l'andamento del terreno, recinto da un muro continuo spezzato nel suo sviluppo tanto da disegnare in modo irregolare una forma quasi ovale, questa risulta interrotta sui punti cardinali da quattro spazi o torri di avvistamento. Il Castello già in epoca medievale era considerato la città capitale dell'isola, trasferita poi nel 1754 ad Argostoli.

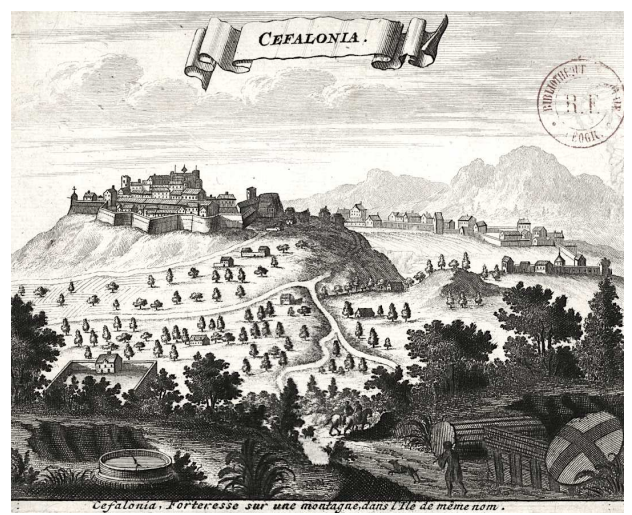

Fig. 2. Cefalonia, incisione [XVIII secolo], BNF Paris.

Nella relazione sullo stato dei luoghi dell'isola di Cefalonia redatta dal Capitano Martin nel 1808 (Fig. 3) vengono descritti minuziosamente i collegamenti fra i diversi luoghi dell'isola mettendo una particolare attenzione nella descrizione dei porti, trascurando però l'efficacia del Forte di San Giorgio dovuta alla sua posizione nel proteggere il porto di Argostoli che nel tempo aveva acquisito sempre più importanza diventando per l'isola quello principale. Nel testimo- niale si racconta e si raffigura il Forte come ancora abitato, con l'ingresso nello spigolo sudovest dove si susseguono in salita una serie di piccole corti passanti che immettono sui camminamenti lungo muro sino a salire, sul lato a nord, nella parte più elevata (AGATP, 1808). Lo spazio centrale è suddiviso in diverse corti delimitate da una ventina di fabbricati che in parte si elevano a tutta altezza e in parte sfruttano le diverse quote per utilizzare spazi interrati, disposti per lo più in linea assecondando i diversi dislivelli, il costruito accoglie "le residenze del comandante, dei suoi ufficiali, i templi, le case nobili, i ma-

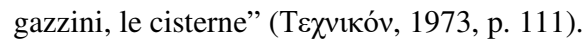

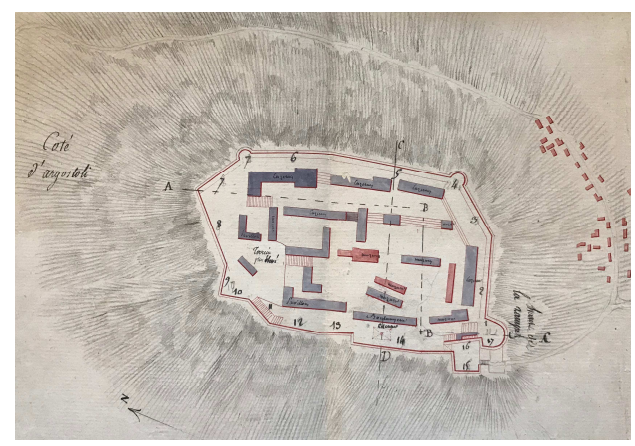

Fig. 3. Martin, Gouvernement des Isles Ionnienne / Direction de Corfou / Plan du Fort St. Georges à Cephalonie, Corfou 1808 (AMDF, 1 VM 68, stralcio).

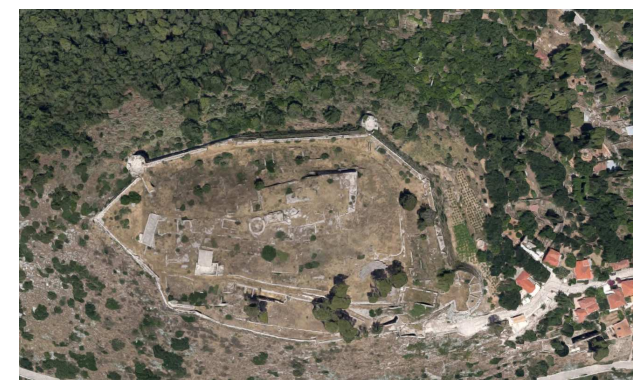

Fig. 4. Forte di S. Giorgio, Cefalonia. Vista aerea attuale.

Il Forte dai francesi viene considerato utile tuttalpiù a proteggere le sole regioni dell'entroterra, quelle disposte a sud non così necessario per le nuove dinamiche infrastrutturali tanto da consigliarne, nel 1813, la demolizione e per: "quanto [riguarda] il sito di St. Georges, che è stato nel frattempo una struttura utile tanto da dargli una 
guarnigione, sono dell'avviso di demolirlo integralmente. Questo perché, anche se su una notevole altura, può essere facilmente colpito dagli attacchi nemici [...]" (AGATP, 1813).

Privo di importanza tattica e senza generare nuovi interessi al suo interno, il Forte è presto abbandonato (Fig. 4).

La consistenza attuale è rilevante soprattutto per la permanenza delle muraglie e dei camminamenti che in parte sono ancora distinguibili dai basamenti murari (tracce al suolo) di alcune costruzioni. Il quartiere interno non esiste più, il sito seppur spoglio si apre al paesaggio come mostrano le antiche incisioni.

\subsection{La Fortezza di Zante (Zakinthos)}

Sulla collina che delimita a ovest la città di Zante s'insedia la Fortezza (Fig. 5). Le mura sono disposte sul crinale in modo irregolare assecondando la morfologia del terreno in forte pendenza, delimitando un ampio sedime di forma triangolare (pari a oltre $90000 \mathrm{~m}^{2}$ ). Il fronte bastionato del settore nord-est risale al XVI secolo a opera dei Veneziani, trattasi nella fattispecie di un intervento volto a migliorare l'assetto tattico dell'intera Fortezza. Su questo lato si susseguono i bastioni Dell'Pozzetto, Minotto, Tre Porte dove insiste l'ingresso dalla Strada Giustiniana e sullo spigolo di nord-ovest il Grimani che incorpora una torre circolare. Le mura dal bastione Grimani proseguono linearmente a est interrotte da due piccoli avamposti semicircolari e dal bastione Bembo da dove si accede direttamente all'area più propriamente dedicata all'insediamento militare; un lungo cammino di ronda conduce allo spigolo sud dove si trovava la torre circolare di san Marco. A metà dello sviluppo delle mura della parete ovest, dall'andamento più irregolare insiste il bastione

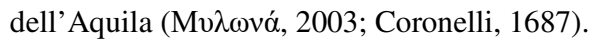

Per l'ampiezza della superficie circoscritta, il Forte accoglie al proprio interno una piccola città con caserme, polverieri e prigioni ma anche chiese, conventi, case, mulino, spazi per la coltivazione, il mercato e approvvigionamento dell' acqua (18 fontane e 4 pozzi), un vero e proprio borgo composto alla metà del XVI secolo

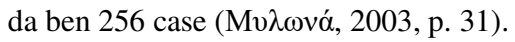

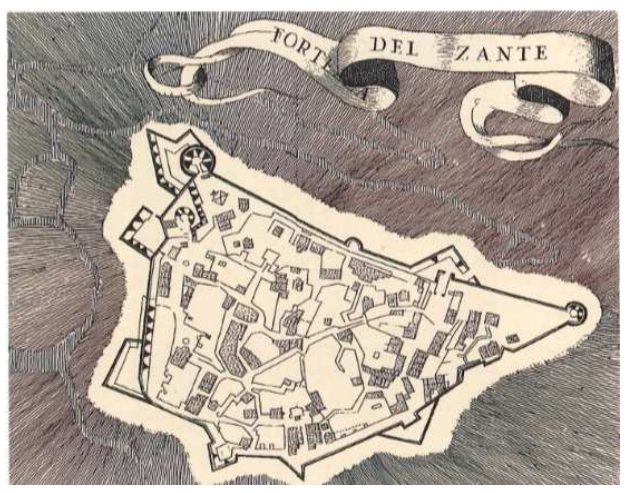

Fig. 5. Fortezza del Zante, incisione (Coronelli, 1687).

La struttura interna di questa piccola città segue la conformazione del suolo con vie tortuose suddivisa in sei distretti S. Giovanni, S. Giorgio, S. Nicola, S. Nikitas, Sta. Laurentia e Sta. Marina e caratterizzata da piccoli nuclei edificati intorno a spazi pubblici, piazze dove all'edificio religioso si affiancano edifici militari e la fontana. Le chiese cattoliche e ortodosse (realizzate fra il XII e il XVII secolo) secondo il rilievo condotto da Salvator e pubblicato nel 1904 ne risultano 12 fra queste le maggiori riguardano i conventi di S. Francesco a sud-ovest posto all'interno del quartiere militare e S. Giovanni a est e la chiesa di S. Salvatore a ovest, sei delle rimanenti sono piccole aule a navata unica che si dispongono ad anello lungo le mura (B. V. Crisopigi, Sta. Barbara, S. Giovanni Teologo, B. V. Laurentiana, Sta. Veneranda, S. Nicola), le altre si situano all'interno del borgo (S. Teodoro, Sta. Marina e S. Giorgio).

Già sul finire del XVII secolo la città di Zante lungomare acquisisce importanza e questo lo mostra il fatto che è "ben habitata da' ricchi Cittadini, da' Mercanti d'ogni Natione (e particolarmente Inglesi, Artigiani, e Marinari), che dal traffico d'Uve Passe, de' Vini, Moscati eccellentissimi, Ogli perfetti, ed altre sorti di frutti ricavano notabili profitti, ritraendone la Repubblica ne' Datj soli ogn'anno grandi proventi. Questi vengono amministrati da un Proveditore, e due Consiglieri Nobili Veneti, che dal Maggiore Consiglio, che regge tutta l'Isola fa la sua Residenza nel Castello fortissimo, che dalla parte di Greco sopra l'eminenza maggiore del monte s'inalza, e dominando tutta l'Isola, difende la 
Città priva d'ogni altra Fortezza" (Coronelli, 1696, p. 180). Nel Forte risiedono principalmente gli Stati Maggiori del Governo e col tempo, con l'affermarsi del porto di Zante e della sua città, risulterà sola residenza dei diversi corpi di armata che si susseguiranno ai Veneziani anche inserendo nuove attività e rifunzionalizzando quanto già di abbandonato (infermeria, ospedale, scuola, biblioteca).

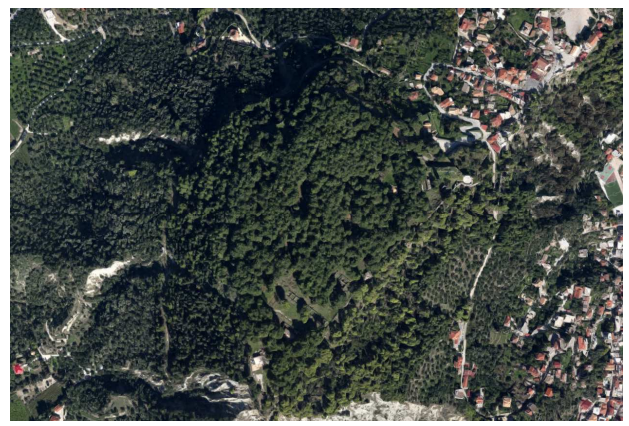

Fig. 6. Forte di Zante. Vista aerea attuale.

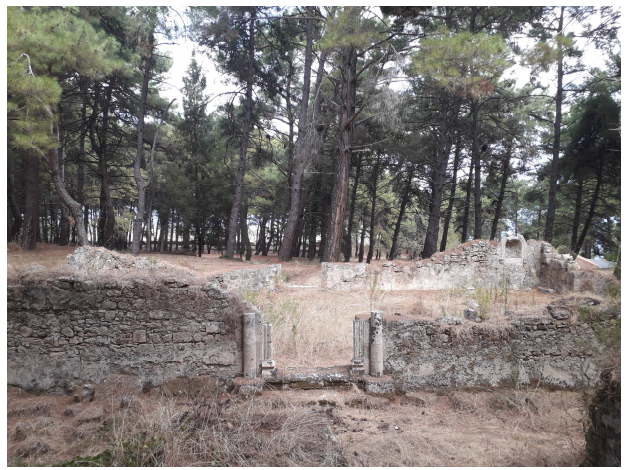

Fig. 7. Il Forte di Zante, resti della chiesa di S. Francesco, stato attuale (Gkrimpa, 2019).

Alle modifiche funzionali e alla dismissione, per Zante, subentrano i grandi terremoti quello del 1893, del 1953 e quello del 1971 che demoliscono l'intero abitato. Attualmente (Figg. 6-8) è possibile riconoscere i sedimi di alcune chiese dal loro tracciato di fondazione: S. Francesco, S. Giovanni Battista, S. Salvatore, B.V. Laurentiana, S. Venerando, S. Giovanni Teologo e alcuni degli edifici militari in elevato: le prigioni e la polveriera, per il resto una folta pineta ha trasformato integralmente il paesaggio, cancellando le antiche strade e i ruderi sono avvolti dalla ve- getazione. La sola permanenza effettiva che disegna la sommità della collina, perché integre, sono le mura e i bastioni che oggi delimitano un grande parco.

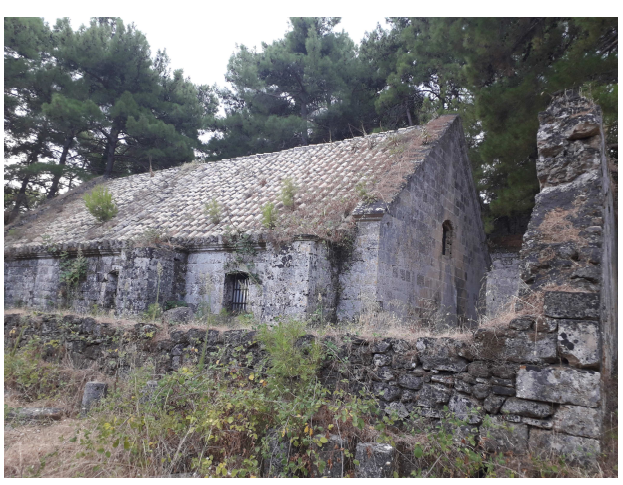

Fig. 8. Il Forte di Zante, resti della Polveriera, stato attuale (Gkrimpa, 2019).

\subsection{Il Forte di Cerigo (Kithira)}

A sud dell'isola, posta su di una collina che sovrasta la baia di Caposali (Kapsali) caratterizzata dalla presenza di due insenature protette dal piccolo promontorio di S. Giorgio, la Fortezza guarda, con il suo Castello, l'isola di Creta governando il passaggio fra il Mar Ionio ed Egeo. Esaminando le incisioni seicentesche del Coronelli la Fortezza è composta da tre settori aventi ciascuna delle proprie mura, il castello a nord, nella parte più alta, accoglie sul perimetro le abitazioni dei soldati e del comandante e al centro la chiesa risalente al XVI secolo dedicata alla Vergine dei Latini che dal 1806 diviene ortodossa, Sta. Myrtidiotissa. Il quartiere militare era poi autonomo ovvero con propria cisterna e magazzini. Al castello si accede attraverso il settore centrale della fortezza che si sviluppa verso sud con ingresso dal borgo esterno dalla Porta Maggiore. La parte mediana è realizzata dai veneziani ed è quella che accoglie la città. In prossimità della porta si localizzano le cisterne con strutture voltate e dopo un breve tratto di strada in salita si entra nella via centrale del quartiere, rettilinea nella sua intera estensione, composta da case a due piani disposte a isolati regolari, è al termine di questa via che si entra, attraverso una porta voltata, al castello. All'interno del settore mediano insistono due chiese, S. Giovanni e Pan- 
toKrator. Il terzo settore è quello posto a valle che avvolge e protegge il Forte lungo il lato est dalla Porta Maggiore al Bastione del castello e l'iconografia (Fig. 9) mostra un quartiere abitato con propria chiesa (Coronelli, 1696).

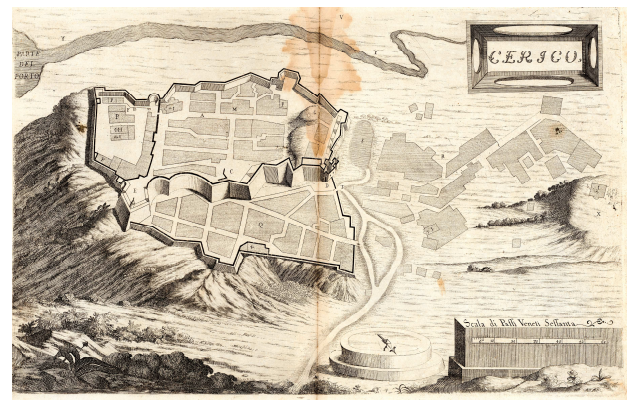

Fig. 9. Cerigo, incisione (Coronelli, 1696).

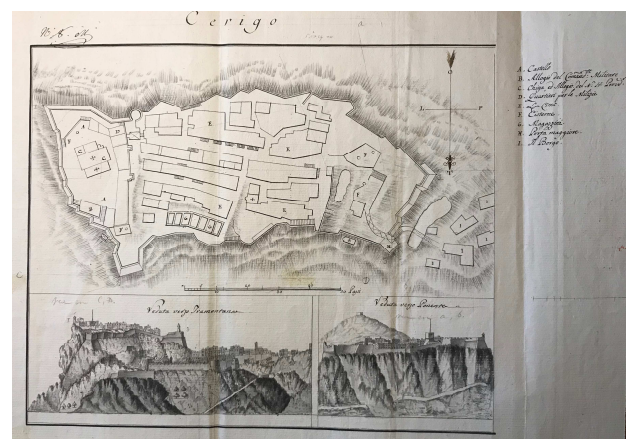

Fig. 10. Plan de la ville de Cerigo avec 2 vues, 1799 (AMDF, 1 VM 68)

La relazione redatta dal Comandante francese delle Isole di Levante Wallonque nel 28 maggio 1798 , che analizza nel dettaglio lo stato dei luoghi, mostra una situazione all'epoca alquanto degradata. (Fig. 10) "Non si può guardare questa fortificazione come a una vera Fortezza poiché manca delle abitazioni solide per la Guarnigione e per i magazzini delle vivande e dell' artiglieria [...] il Fronte dove si trova l'Entrata a contatto con il Borgo dove all'interno è formata da una Facciata frantumata con due semi Bastioni [dove] manca il parapetto e la piattaforma per mettere una batteria [...]. Le abitazioni non sono in buono Stato, una Guarnigione di 80 uomini avrebbe difficoltà a mettersi al riparo. Il Corpo di Guardia del quartiere e del Comandante, detto Palazzo Pubblico, manca del cosiddetto Campo;
[...] Il quartiere detto della Grande Guardia alla porta di ingresso non è più abitabile per il cattivo Stato delle coperture, dei letti da Campo e della porta" (AGATP, 1798). È chiaro dalle descrizioni di fine Settecento che il Forte non è più adeguato alle nuove richieste tattiche oltre che abitative. Attualmente il Forte è al suo interno quasi integralmente spogliato dai suoi edifici lasciandone traccia con tratti di muro e qualche facciata; mentre è definibile il tratto di mura esterne del settore centrale, non si percepisce più quello che un tempo era la divisione fra città e castello (Fig. 11).

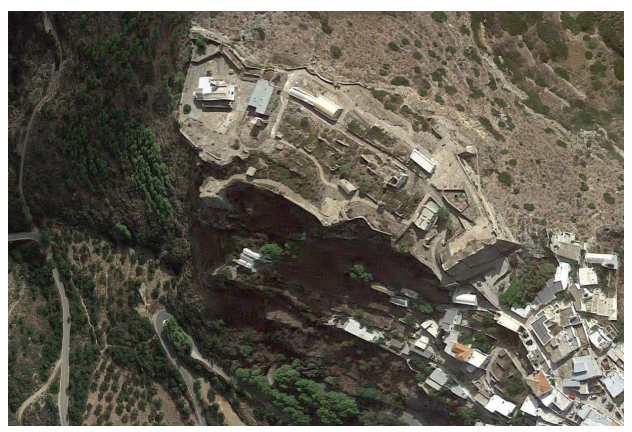

Fig. 11. Forte di Cerigo. Vista aerea attuale.

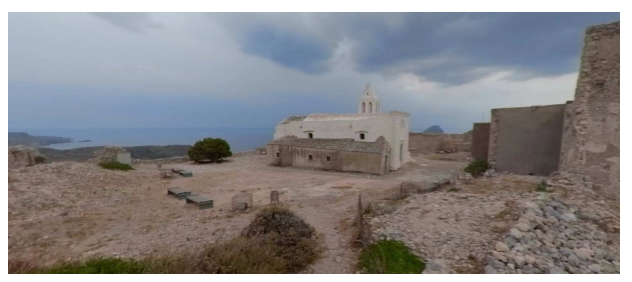

Fig. 12. Forte di Cerigo. La chiesa ortodossa Sta. Myrtidiotissa nella parte del Castello. Stato attuale.

A differenza degli altri siti analizzati in questo caso permangono alcuni edifici isolati (le due chiese, le cisterne, parte del quartiere delle milizie) che permettono ancora di far emergere l'organizzazione distributiva delle antiche strade e la localizzazione dei servizi (Fig. 12).

\section{Per un progetto di valorizzazione}

$\mathrm{Nel}$ configurare un progetto di salvaguardia per le Fortezze delle Isole Ioniche occorre innanzitutto inserirle nel proprio contesto nel valutare problematiche e risorse. La situazione demografica e turistica appare molto varia, anche se tutte 
le isole sono dotate di proprio aeroporto e approdo portuale di linea, è evidente come le componenti ambientali e le scelte politiche intraprese negli ultimi decenni a favore del turismo delineino un quadro eterogeneo.

Se si confrontano così i dati demografici nel decennio 2001-2011 è possibile constatare che l'aumento della popolazione è strettamente correlato con l'incremento delle attività turistiche, con un $+3 \%$ per le isole di Corfù, Cefalonia, Zante. Dall'esamina dei dati relativi agli arrivi turistici 2010-2018 (INSETE, 2019) emerge, nel caso di Zante, un aumento senza precedenti, tanto da far crescere i suoi arrivi del $70 \%$ (ELSTAT, 2019) con turisti che per il $92 \%$ provengono da altre nazioni. Il dato è veramente importante se si pensa che la popolazione residente è oggi pari a 40758 e che nel mese di agosto i turisti quintuplicano la residenzialità con una crescita di ulteriori 200000 persone. Un aumento sostenuto da alcune azioni prima fra tutte l'incremento delle strutture alberghiere, raddoppiate dal 2010, se ne contano ben 301 nel 2018 (INSETE, 2019). Una politica che non ha considerato però la modalità di svolgimento delle attività che si sarebbero insediate, preferendo la quantità alla qualità, e che spesso non è riuscita a colmare i servizi di sostegno, necessari a un vivere armonico.

Per verificare come i turisti impiegano il loro tempo di vacanza e se c'è interesse nei riguardi delle visite museali o alla partecipazione di attività culturali si possono analizzare i dati riguardanti gli ingressi di visita alle singole Fortezze che se risultano strettamente proporzionali ai flussi turistici delle singole località, è ugualmente evidente l'interesse dove insistono programmi di valorizzazione. Verificando che tutte le Fortezze sono inserite nelle guide turistiche come luogo di visita, ovvero considerate quali spazi di interesse storico, il maggior afflusso è da segnalarsi dove insistono anche altre attività come a Cefalonia dove è il 20\% (pari a 31083 i visitatori) dei turisti a visitare il Forte S. Giorgio. Si può constatare che il dato è sostenuto dall'attività intrapresa dall'Eforato all'antichità di Cefalonia e Itaca, attraverso il progetto "Kó $\sigma \tau \omega \omega v$

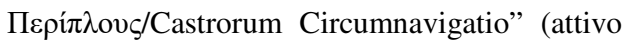
dal 1998), finalizzato al coinvolgimento del For- te per eventi pubblici, per questo al suo interno, in tempi recenti, è stato inserito un piccolo spazio teatrale dove si svolgono spettacoli all'aperto contribuendo a sostenerne l'interesse. Il progetto riguarda anche l'avvio a studi scientificidocumentari sull'origine e le trasformazioni del Forte oltre a prefigurare azioni di miglioramento nella fruizione degli spazi, ovvero nell'accessibilità e sviluppo dei percorsi di visita e dotazione di servizi, come anche prefigurare l'utilizzo di nuove tecnologie per agevolare la

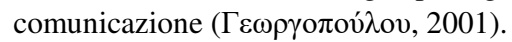

Se si opera ora un confronto con le altre isole emerge che se per Leucade i visitatori risultano pari all' $11 \%$ dei turisti il dato si abbassa notevolmente sino al $2 \%$ per Zante a prova di un diminuito interesse quando gli spazi non accolgono altre attività complementari che possano interessare anche i residenti. L'attenzione per le Fortezze, nel trascorrere mezza giornata del proprio soggiorno in questi luoghi dove il paesaggio entra fortemente in interazione, è comunque alta, superando in alcuni casi la visita ai Musei, come a Zante dove se i visitatori al Forte nel 2018 risultano 14.127 al Museo archeologico e della Città gli ingressi diminuiscono a 9515, stessa sorte per Corfù, la visita ai castelli ha riguardato 185135 persone mentre per i musei se ne contano 61686 (2018). Escludendo però la realtà di Corfù, in quanto la fortezza (Castello Vecchio) è parte costitutiva dell'iscrizione della città al $\mathrm{Pa}$ trimonio UNESCO e dove la visita dell'opera è integrata alle attività Museali e Archivistiche della città contabilizzando una media annuale degli ultimi dieci anni di 135630 di visitatori, è immediato constatare confrontandolo con le altre Fortezze che queste hanno un ruolo decisamente marginale. Prima fra tutte è proprio Zante che arriva a un punto di saturazione a riguardo dell'offerta turistica, tale da necessitare, oggi, di ripensare al modello a cui riferirsi. Elementi di forza quali la durata media di permanenza del turista che è pari a 7 giorni (a differenza delle altre isole dove la permanenza risulta minore) e la presenza di un Parco Naturale Protetto, induce a pensare che sia possibile proporre un turismo più sostenibile interessato e dedicato alla cultura che il luogo propone. Il Forte quale ampio spazio può essere partecipe nel accogliere attività ludi- 
co-ricreative ma soprattutto didattiche che coinvolgano i residenti e siano una premessa di conoscenza culturale per i turisti. Proporre passeggiate a tema fra $\mathrm{i}$ ruderi anche per beneficiare della natura disegnando percorsi narrativi dove, le tracce degli edifici, rimandino informazioni sulla vita di un tempo o su come oggi rispettare il patrimonio anche attraverso le coltivazioni come quella dell'ulivo e la produzione dell'olio, sono fra le possibilità del ri-abitare la cittàfortezza.

\section{Conclusioni}

Nel costruire scenari attualizzabili per la valorizzazione dei Forti delle Isole Ioniche, si è valutato l'apparato storico-documentario, la consistenza attuale, l'interesse culturale delle opere e i progetti in corso. Alla situazione relativa allo stato dei luoghi molto critica dipendente dall'abbandono nel tempo di questi siti e al degrado diffuso delle strutture, si affiancano i dati relativi alle visite da parte dei turisti che mostrano delle potenzialità nel prevedere di investire nuove risorse. Alla necessità per tutti i Forti di un aggiornamento nei riguardi dell'accessibilità e comunicazione della fisionomia urbana (cartelloni/impiego tecnologie per visite virtuali) è evidente il bisogno di far interagire questi beni con l'esigenza ormai imminente di prefigurare nuovi spazi a sostegno di un turismo sostenibile con attività che vadano nella direzione di una salvaguardia diffusa del patrimonio, del paesaggio e della cultura locale.

\section{Note}

Nel testo si fa riferimento alla documentazione contenuta presso gli Archives de la Guerre et de l'Armée de Terre, Paris - AGATP, Génie Fortifications Dépôt des fortifications, faldone $1 \mathrm{VM}$ 68 (Cefalonia e Cerigo), 1 VM 296 (Zante); per le incisioni del Coronelli si rimanda anche alla Biblioteca Nazionale Marciana, Venezia, cartella 225.d.15 (isole di Cefalonia, Cerigo e Zante). I siti consultati per i dati statistici: ELSTAT, 2019 http://www.statistics.gr; INSETE, 2019 http:// www.insete.gr

\section{Bibliography}

Ciriacono, S. (1997). "L'olio a Venezia in età moderna. I consumi alimentari e gli altri usi”, in Cavaciocchi, S. ed., Alimentazione e Nutrizione, secc. XIII-XVIII, Le Monnier, Firenze. pp. 301-312.

Coronelli, V.M. (1687). Description géographique et historique de la Morée, reconquise par les Vénitiens, du royaume de Négrepont et d'autres lieux circonvoisins [...], Paris.

Coronelli, V.M. (1696). Isolario descrittione Geografico-Historica, sacro-profana, antico-moderna, politica, naturale, e poetica. Mari, golfi, seni, piagge, porti [...], Venezia.

Moroni, M. (2013). "Produzione e commercio del sapone nel Mediterraneo tra basso medioevo ed età moderna", in Stefano, E. Di., ed., Produzioni e commerci nelle province dello Stato pontificio. Imprenditori, mercanti, reti (secoli XIV-XVI), Quaderni monografici di Proposte e ricerche, 38. pp. 140-154.

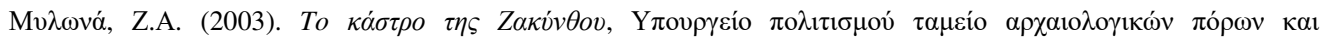

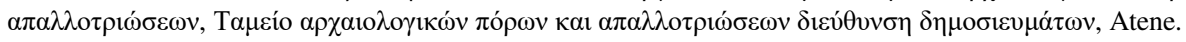

Salvator, L. (1904). Zante, Druk und Verlag von Heinr, Prague.

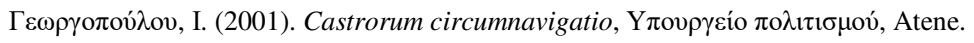

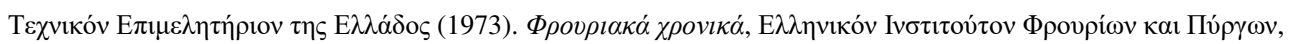
Atene. 